organs. How does it come to pass that in autumn the leaves of some of our forest trees exhibit a brilliant livery of crimson, while others exhibit only a yellow or golden glory? Take, for instance, the case of the ash constituents in the dry substance of the leaf. It is known by analysis that the percentage of ash increases through nearly the whole life of the leaf in beech, sycamore, elm, but not in oak, Jarch, cherry, \&c. ; it depends a good deal on whether some one ash constituent (generally lime or silica) is being steadily stored up. For example, the dry leaf of Acer campestre on May I has 6 per cent. ash, and in October 16.2 per cent. ash; the dry leat of Prumus avium has on April $28,7.8$ per cent., and on October $2,7.2$ per cent. ash. Now the leaf of the former tree is only yellow in autumn and never red, while that of the latter is very often beautifully crimson. In the former case there is a kind of gradual decay or death of some of the cells (mostly of the upper epidermis) which occasions a drainage of mineral and organic substances to these parts from the still living tissues. This drainage and accumulation attest, in facl, such a decay; and what is more, they seem to have a distinct influence over the ultimate autumnal coloration of the leaf itself. It is easy to understand, in fact, that the leaves which exhibit such a decay and approach to dissolution are just these wherein the chromogen precursive of the brilliant red coloration would likewise suffer an analogous kind of change, i.e. it would tend to become brown, to produce phlobaphene, just as it does in the outer bark which is the practically dead portion of the rind. Where this accumulation of mineral matter and all which it implies does not take place, as in cherries, currants, American oaks, pears, wild vine, barberry, \&c., then the chromogen does not deteriorate; it evolves its proper pigment, and assumes the flush and glow of active living colour. On the other hand, in elms, chestnut, linden, birch, poplars, \&c., which are never red but only yellow, it is only the vivid carotin attached to the last faded and now exhausted chlorophyll which gleams forth, but only fur a time, and if not too much obstructed by the dull browns of decomposed carbohydrates and superoxidised tannic chromogens.

Patterdale, Westmorland.

\section{Homochronous Heredity and Changes of} Pronunciation.

SeEING that in ancient German, or rather Gothic, Swedish, Danish, probably in French, and possibly in Sardinian, the th sound surviving in English (though much less frequent than it used to be) was once largely used, but nowadays Frenchmen and Germans find a difficulty with it, I should like to know whether systematic experiments have been made as to whether children of various ages of these two nationalities can pronounce it more exactly and spontaneously than their compatriots of a maturer age? I should like to make the same inquiry concern. ing English children and their pronunciation of the gutturals discarded or altered in such words as night, bough or laugh?

23 Upper Bedford Place, W.C.

Charles f. STUarT-Menteath.

Authorities:-Helfenstein, "Comparative Grammar of the Teutonic Languages," I870, pp. 156-9; G. Koerting, "Neugriechisch und Rnmanisch," I896, p. 23; W. Meyer-Luebke, "Grammatik der Romanischen Sprachen," I 890, p. 428.

The Daylight Meteor of Sunday, September 2.

As Mr. Denning expresses a wish in your issue of September $I_{3}$ for further information concerning this meteor, I write to inform you of what I saw myself.

I observed the time at which the meteor fell, and made it 6.50 , but my watch is no chronometer. I saw the meteor from the road, between Deganwy and Llandudno, and it appeared to fall over the Little Orme's head. If you join this point to Leyburn in Yorkshire, you have the line as near as I can give it, and I do not think it is very far out. I did not note any column of smoke or cloud after the meteor fell. Its path was vertical. Some one says its angle of appearance was $35^{\circ}$, and disappear. ance $25^{\circ}$, which I should say is about correct. The sun was shining brightly, though low down in the west. The brilliance was greatest just before disappearance. I have never before seen any meteor to compare with it in brighiness.

38 Hillfield Road, Hampstead.

T. ROOKE.
THE meteor of September 2, described in your issue of September 13, was seen in Ireland also, in even brighter day. light.

I noted the time, 6.27 p.m. (Irish), and the direction, E.N.E., from a point near Enniskerry, co. Wicklow. There was a possible error of a couple of minutes in my watch, and a con. siderable error possible in the estimated direction, which was a rough approximation made without a compass.

\section{B. ST. G. Lefroy.}

\section{THE THEORY OF IONS.}

FVER since Faraday enunciated the law of electrolysis, that the same quantity of electricity passed when chemically equivalent masses of different substances were produced, it has been a matter of speculation whether this may not be due to atomic charges of electricity. Every one, in describing electrolysis and explaining how the substances evolved appeared at the electrodes without any apparent action in between them, based his description and explanation upon the supposition of electric charges on the atoms. Some substances, such as hydrogen, were given positive, and some, such as chlorine, were given negative charges, and the electric current through the liquid was explained as due to the convection of these charges by the moving atoms or groups of atoms, and the movements of these were ascribed to the electric force acting on these charges. The amount of the charge on each atom or group of atoms was proportional to its valency, and as this has with good reason always been taken as a whole number, the charges ascribed to the moving elements were all simple multiples of the charge ascribed to a monovalent atom, such as hydrogen or chlorine. All this has naturally led to the hypothesis that electricity itself is atomic. In electrolysis, at least, there is a certain minimum quantity that corresponds to a single atomic bond, and quantities of electricity transferred by electrolysis are always multiples of this unit. It was surely natural, then, to give a name to this important physical unit quantity of electricity, and it has consequently been called an "electron."

Further, in electrolysis, the electrons always appear connected with, and travelling with, certain atoms or groups of atoms. For example, in copper sulphate solutions, the positive electrons travel in pairs with the divalent copper atoms, and the negative electrons with the divalent atomic group $\mathrm{SO}_{4}$. These charged atoms, or groups of atoms, playing such an important part in electrolysis, have been called "ions."

Now there is a very important difference between different liquids in their behaviour when we try to pass an electric current through them. Some are quite easily decomposed, others offer a very great resistance, and it has been a matter of most interesting speculation as to the cause of this. In the first place, most of the easily decomposed liquids are solutions in water, of acids, alkalis or salts, and this has naturally attracted attention. In the second place, these solutions are all ones in which double decompositions, and such-like chemical actions take place with facility. Can a common explanation be given of this remarkable coincidence of electric conductivity and chemical activity? Electric conductivity is due to two causes - first, the electric charges on the ions; and second, the independent mobility of these oppositely charged ions under electric force. Without entering upon the very interesting questions involved in innumerable speculations as to the causes of these charges and of the mobility of the ions, all modern theories acknowledge that, in some way or another, water, and some other liquids in a less degree, have the very remarkable property of conferring upon certain substances dissolved in them the wonderful independent mobility of the ions which we see in electrolysis.

No. I6I 3 , voL. 62 ] 
In consequence of this mobility of these differently electrified ions, it is easy to understand their chemical activity in these conducting solutions, and thus these two important properties of these solutions receive $a$ common explanation. No really satisfactory explanation of how the solvent water confers on substances dissolved in it this wonderful independent mobility of the ions has yet been proposed. Some writers have described the phenomena as if all that was needed was to assert that the ions move about independently, that the material, $\mathrm{CuSO}_{4}$ for example, is simply dissociated into $\mathrm{Cu}$ and $\mathrm{SO}_{4}$, and that these ions gad about freely and independently in the liquid. Such writers consequently speak of the substance as being dissociated in solution. In what is recognised as ordinary chemical dissociation there is no different electrification of the constituents into which the substance is dissociated, and there is thus an essential distinction between the independent mobility of electrified ions in solution and what is recognised as chemical dissociation. Whatever be the true account of the matter, it is almost certainly very much more complicated than ordinary chemical dissociation, and the action of the water is evidently of the first importance. There is great difficulty in explaining this independent mobility, on account of two things which have not been satisfactorily explained as yet. In the first place, it is very hard to understand why these oppositely electrified ions do not combine together in pairs as they would do if they were merely under the action of the electric forces due to their opposite electri. fication. In the second place, it is very hard to understand where the energy comes from that is required to separate these independent ions and keep them free from one another. When copper sulphate is dissolved in water there is very little change of temperature; if it be the anhydrous salt, there is a rise of temperature; while we would naturally expect an enormous absorption of heat to account for all the energy that would be required to separate the $\mathrm{Cu}$ and $\mathrm{SO}_{4}$ from one another. This all shows how very different this phenomenon is from ordinary dissociation, and in consequence this peculiar action of water, and in a less degree of some other solvents, has been called "ionisation." The two most important properties of an ionised fluid are conductivity for electricity, and a remarkably chemical activity which has been shown to be directly proportional to the conductivity.

Besides liquids there are gaseous conductors of electri. city. Gases do not usually conduct electricity at all. Even under circumstances when one would naturally expect them to carry electric charges on their molecules, they seem quite incapable of doing so. When the surface of a liquid is electrified and it evaporates, one would naturally expect the escaping molecules of gas to carry away with them some of the electric charge from the surface of the liquid. It is not known whether an electrified metal volatilising would or would not carry away with it some of the superficial electric charge, but ordinary liquids volatilising certainly do so to a very small extent, if at all. This may be, of course, because the charge is carried by superficial ions, and it is not the ions that escape, but the molecules of the liquid itself. But why these extremely morable ions cannot escape as a gas from the surface of the liquid is a matter still requiring explanation.

Under a great variety of circumstances, however, gases are able to conduct electricity. Leaving aside the spark, glow and arc discharges in gases at high pressures, and the well-known discharges in gases at low pressures, in all which cases there is evidently something like a breaking up of the gas itself under intense electric force, there are a number of cases in which a gas can conduct electricity under quite feeble electric forces.
Within any space at all close to a spark discharge of any kind, in flames and in the gases escaping from them, in the neighbourhood of surfaces of solids illuminated by ultra-violet light, in the neighbourhood of surfaces of solids being acted on chemically by the gas, in a gas traversed by kathode rays, and in a gas traversed by $\mathrm{X}$-radiations and by those various, most curious and remarkable radiations that have been classed under the name of Becquerel rays - in all these cases gases conduct electricity, apparently quite freely.

Under these circumstances it has been usual to describe a gas in this condition as "ionised" and to seek for a separate and independent mobility of its ions. Great success has attended these investigations. The difficulties that surround ionisation in liquids are mostly absent here. The ions, if left for a time to themselves, do combine together in pairs, and it requires a continu ous and considerable expenditure of energy to keep them apart. The diffusion of the independent electricities has been studied, and many quantitative results that were to be expected from the theory have been proved to exist.

There is, however, an important difference between the conductivity of a gas and of a liquid. In the case of a liquid, the electricity always travels along with matter in the form of an atom or a group of atoms; in a gas there is every reason to believe that we are often dealing with electric charges which, if connected with matter at all, are connected with masses which are about 500 times smaller than a hydrogen atom. So far no good reason has been given for believing that the electric charges that move about among the molecules of a gas carry any matter along with them. There does not seem any difficulty in supposing that the electric charge of an atom can exist independently of the atom. All theories of electrolysis have supposed that electric charges are transferred within the liquid along with material atoms, but from the liquid to the electrodes all theories have supposed that the electric charges jump from the liquid atom to the electrode; and if it can jump from one atom to another, there seems no reasonable objection to believing in its independent existence. On account of the difference between the nature of the conductivity of a gas and of a liquid, it would be well to confine the term ionisation to the case of conductivity due to the mobility of charged atoms or groups of atoms, and to call the conductivity due to the existence of these mobile electric charges which are not connected with atoms by another name, such as "electronisation."

One of the most remarkable results of the study of these mobile electric charges which are unconnected with atoms is that only negative electric charges have as yet been discovered to be free from atoms ; the corresponding positive charges seem to be always attached to atoms or groups of atoms. This has naturally led to a rehabilitation of the old single fluid theory of electricity in which matter plays the part of the positive fluid in the old double fluid theories, and the phenomena of electronisation, so far known, certainly lend support to this hypothesis. But we really know so little about the subject, that it is rather too soon to form anything beyond a rough working hypothesis. So long as we know that there exist outstanding, second order effects like gravitation, we are premature in concluding that the connection between positive electrification and matter proves any first order difference between positive and negative electricity, as it may be a second order effect.

The conductivity of gases produced by the pressure of these movable electric charges is in some respects more analogous to that of metals than to that of liquids. In liquids an electric current is accompanied by streams of matter, while in the electronised gas, so far as it has been No. I6I3, VOL. 62] 
observed, there may be no stream of matter attached to the electric charges which carry the current. It has consequently been suggested, and with good reason, that in solids and melted metals, which conduct metallically, the electrons are freely movable, and that this is the cause of their conductivity. There is some reason to believe that in this case also it is the negative electron which is most freely movable. Some most interesting calculations have been made upon this hypothesis, in which it has been supposed that there was something like a gaseous pressure of these mobile electrons in the metal. Thermoelectric effects have been attributed to the dependence of this pressure upon temperature, and the convection of heat accompanying electric currents has been attributed to the convection of energy of irregular motion by these electrons. The Hall effect has also been shown to be a possible consequence of a different mobility of the positive and negative electrons.

Upon these principles it is natural to attribute the magnetic properties of iron and other substances to electrons describing orbits round the atoms. These revolving electrons, in this case, represent the amperean atomic currents to which magnetisation has long been attributed. A remarkable confirmation of this has been derived from the Zeeman effect, which can be explained by the supposition that negative electrons are describing orbits round the atoms. Further, the mass that moves with these electrons has been shown to be of the same order of magnitude as the 5ooth part of the mass of an atom of hydrogen, which, from experiments on gaseous electronisation, seems to accompany the free electrons in a gas when it conducts.

There seems to be some reason to think that in a highly magnetisable material, such as iron, either there are more than the four electrons corresponding to its atomicity in rotation, or else that these are rotating very much more rapidly than corresponds to the vibrations of ordinary light. Some objection may be taken to the latter hypothesis from the difficulty of explaining why enormously rapid ether waves are not thereby generated in the surrounding medium, and the energy of the motion thereby lost by radiation. There are suggested explanations of this difficulty, but the other hypothesis, that matter has in it many more electrons than correspond to its atomicity, and that these latter are merely peculiar in being removable, agrees with a very interesting suggestion that all matter is built up of electrons. That an atom of hydrogen, for example, consists of some 500 electrons, one of oxygen of some 8000 , and so forth. This is a natural deduction from these speculations, and receives some confirmation from its being consistent with the change in dimensions of a body as it moves in different directions through the ether which has been assumed in order to explain the experiment on the motion of the earth through the ether, which Michelson and Morley conducted. A supposition such as this naturally suggests that atoms could be built up of electrons as well as the electrons separated from matter; and if that be so, there seems no impossibility in the dreams of the alchemist, and an element of one kind may some day be transmitted into that of another. What is as yet known is, however, a very slender foundation for these speculations, and it is quite likely that matter and electricity are distinct in kind, and cannot be transmuted into one another in the way suggested.

Enough has been said in this very sketchy description of ionic theory to show how far-reaching it is; how it touches upon the confines of our knowledge and upon the borderland between physics and chemistry. Advances in our knowledge of ionic theory are likely to dispel many of the clouds surrounding the connection of matter and ether, and may lay the foundations for an intelligible structure of the physical universe.

G. F. F. G.
THE RECENT CRETAN DISCOVERIES AND THEIR BEARING ON THE EARLY CUL. TURE AND ETHNOGRAPHY OF THE EAST MEDITERRANEAN BASIN.

WHILE recently excavating the prehistoric Palace of Knossos, which lies in the great central gap between the higher ranges of Crete, mid-way between the peaks of Ida and Dicta, I was much struck by the almost continuous dualistic style of the elements. But in this case the "eternal struggle" was not between East and West. It was North and South that here fought it out. The boreal blasts which have collected from the steppes of Eastern Europe sweep almost unopposed across the Eyean, and find their first obstacle in the long mountain wall of Crete. They pour through the central gap. Not unopposed, however; they are beaten back, and their place triumphantly taken for weeks at a time, by the parching South wind-the Notios of the Cretan natives - which is really the Khamsin of the Libyan Desert. Owing to the fact that the shoot and dumping-ground of the excavations was, perforce, at the southern end, the works were interrupted for days at a time by an overwhelming dust-cloud due to this cause, for the Khamsin seems to have an affinity for dust out of proportion to its actual strength. Disagreeable, however, as were these hindrances to the work of the spade, one had at least leisure to reflect on the historic lessons supplied by these natural phenomena. Crete certainly stands geographically in closer relation to Asia Minor than it does to Africa. Carpathos and Rhodes, not to speak of minor islands, afford natural stepping-stones of intercourse. The actual relations between Crete and Anatolia, ethnic and other, must not be underrated. Yet in a broad historic point of view Crete stands apart from it. It was not like Cyprus, which, although at different times it has become an outpost of Egypt and of Europe, has always remained essentially a part of Western Asia. But the main currents of Cretan history, like those of its two prevalent winds, have been Northern and Southern-European and African. Of its two direct geographical connections, that with Greece and that with Anatolia, it has consistently held to the former. On the other hand, its intercourse with the opposite Libyan coast-the Cyrenaica-and with Egypt has been singularly continuous from a very remote period. And in this lies the high importance of the part played by the island in the early history of European culture. Germs received here from the Nile Valley and its borderlands, at a time when the greater part of Europe was still in its Stone Age, were propagated northwards and westwards, and seedlings hence derived spread in prehistoric times, and by more than one channel, as far as the British islands.

During five successive campaigns of preliminary exploration in Crete, I was able to collect a variety of evidence establishing the very early derivation of certain indigenous forms of stone vases and decorative motives from those of Egypt. A series of archaic Cretan seals exhibited designs copied almost directly from those of Twelfth Bynasty scarabs, and approximately dating, therefore, from the middle of the third millennium before our era, while steatite vases were found almost indistinguishable in form from Old Empire types of considerably earlier date. The primitive three-sided seal-stones, on which appear the first rudiments of Cretan script, reproduce the type of a three-sided seal, apparently of Libyan origin, which, from its analogy with a special class of Egyptian cylinders, approximately date from the middle of the fourth millennium B.C. So long, however, as the early archæological strata of the Cyrenaica are left as at present wholly unexplored, a great blank is still left in the materials for comparison on the Libyan side. It

NO. I 6 I 3 , VOL. 62$]$ 